\title{
A BOND GRAPH APPROACH TO ANALYSIS AND SIMULATION OF A COUPLED TORSION BEAM AXLE
}

\author{
D. Jordan McCrone \\ Donald Margolis \\ Mechanical and Aerospace Engineering \\ University of California, Davis \\ One Shields Ave. \\ Davis, CA 95616
}

\author{
Namho Kim \\ Hyundai Motor Company Division \\ Hyundai Motor Company \\ 150, Hyundaiyeonguso-ro, Hwaseong-si, Gyeonggi-do \\ 18280, Korea
}

\begin{abstract}
Coupled Torsion Beam Axles (CTBA) are combined with springs and dampers to form suspensions for compact automobiles. These are large metal components with complex geometries, presenting difficulty in estimating their influence on vehicle dynamics. Due to this complexity, the design process involves generating Finite Element Models (FEM) for analysis and global vehicle performance. However, FEM analyses are time-consuming. This paper presents, as an alternative approach, a low-order model for simulation developed from first principles. The model is simulated for common dynamic and static tire/axle deflection conditions and compared to real vehicle Suspension Parameter Measurement Device (SPMD) results. The trends of the dynamic model and actual vehicle test data are presented and compared.
\end{abstract}

Keywords: Vehicle Dynamics, Kinematics and Compliance, Bond Graphs

\section{INTRODUCTION}

Coupled Torsion Beam Axle (CTBA) suspension types have been a popular choice among automakers since they were first introduced by Volkswagen in 1974 (Crolla, Foster, Kobayashi, and Vaughan 2015). CTBAs are traditionally used within the rear suspensions of compact, A-, B-, and C-segment vehicles. Reasons for their incorporation include their low cost and convenient packaging due to reduced geometric profiles.

A diagram of a CTBA suspension can be seen in Figure 1. The CTBA combines trailing arms and a twistbeam to form an H-configuration. The assembly is welded (or otherwise connected) to form a solid member. The twist beam is a variation of a hollow tube with circular cross-section and dimensioned to provide a desired compliance allowing for angular displacements about multiple axes between the left and right trailing arms. Rigidly integrated into each of these trailing arms are the respective wheel hubs.

The study of these systems is important because suspension deflection from applied forces results in vehicle steer characteristics (through displacements in wheel toe and camber) that impact global behavior. This behavior may contribute to oversteer conditions that can make the vehicle unstable. 


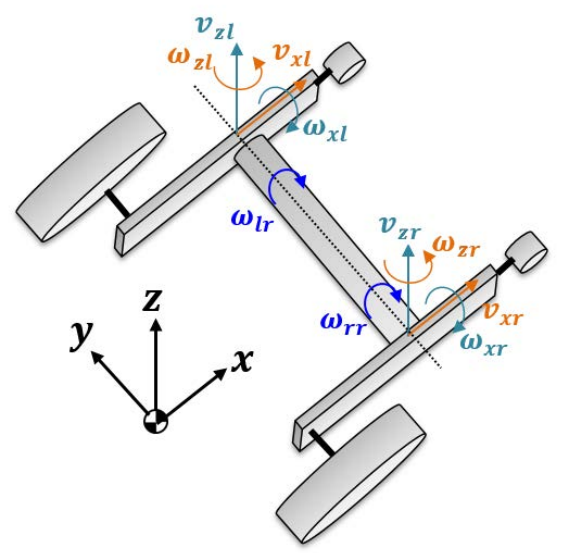

Figure 1: Diagram of CTBA suspension component where trailing arms with transverse torsion beam are clearly visible.

In developing a new model, a design team will generate a Finite Element Model (FEM) to generate Kinematics and Compliance (KC) curves. These $\mathrm{KC}$ curves help designers determine global vehicle performance and stability. By applying forces and moments that correspond to a specific suite of common vehicle modes, the KC curves will suggest trends that indicate stable or unstable behavior. For example, KC curves will be generated that show toe-in and -out characteristics resulting from vehicle body roll; if the outer wheel displays excessive toe-out during a roll, the designers may wish to adjust bushing stiffnesses and torsion beam properties accordingly.

FEM is a time consuming and computationally expensive process. When integrated into the design process, which is inherently iterative, FEM introduces bottlenecks to new model development. Therefore, an alternative approach is developed using Bond Graph dynamics modeling techniques. Bond Graphs (discussed in more detail in Section 2) represent a graphical modeling tool which acts as an intermediate step between the modeling decisions that produce a system diagram and the generation of mathematical equations describing the dynamic system's behavior. The inherent modularity of Bond Graphs allows for changes to be quickly made to design parameters or model complexity; these changes are systematically propogated to the set of first-order differential state equations describing system behavior.

Hyundai Motor Company incorporates the CTBA suspension-type into their value-edition vehicles, including the Elantra and the i30. With the ability to design simulation experiments quickly and concurrently with FEM analysis, FEM results can potentially be demoted to a validation procedure such that vehicle design may proceed primarily based on the first principles model simulation results. The information garnered from static and dynamic simulation using a low order model may suggest compliance values in the bushing selection process, even before a FEM study has been completed.

\section{BOND GRAPH MODELING}

A quick introduction to Bond Graph modeling is presented to describe the model presented in Section 3. A more general description can be found in (Karnopp, Margolis, and Rosenberg 2012). Bond Graphs are graphical representations of system diagrams that allow an engineer to programmatically generate the system state equations. These state equations will be produced in first-order derivative form, which allows them to be readily ported into simulation. Bond Graphs have an added benefit that they can easily bookkeep the power transferred across energy domains and display it in a single Bond Graph; an interesting side-effect 
of this is that studying a bond graph doesn't necessarily reveal exactly whether the Bond Graph represents a mechanical, electrical, hydraulic, chemical, thermodynamic, etc. system.

Bond Graphs consist of a basic set of features. Among these are the eponymous "bonds," which represent power flowing between elements. There are always two variables, called power variables, that are associated with a bond: an effort and a flow. The product of these power variables results in units of power. For example, in a translatory mechanical system, the product of a effort (force, $N$ ) and a flow (velocity, $\mathrm{m} / \mathrm{s}$ ) results in a unit of power $(W)$; as do, in rotary mechanics, the product of torque and angular rate.

Additional features of each bond are the assignments of power convention and causality. A power direction assignment selects the direction that assumes positive power transfer given the product of positive-valued power variables. This is represented by a half arrow at the end of the bond (examples in Figure 2). Causal relationships are represented by a short, perpendicular "tick" mark appended to the end of a bond. If hypothetical elements A and B are connected by a bond, and element A is exerting an effort on element B, then element $\mathrm{A}$ will be receiving a flow as a result of their mutual interaction. One could equivalently describe that element B sets a flow onto element A, and in so doing receives an effort from element A. Bond Graphs do not discriminate between these descriptions.

Other features of Bond Graphs include Inertance and Compliance elements, which bookkeep energy storage; i.e., in mechanical systems, masses and springs, respectively. Each of these elements are represented by a constitutive law for energy storage. For inertance, the flow coming out of the bond is a function of mass momentum (the integral of the effort). For compliance, the effort the element exerts on the system is a function of its displacement (the integral of the flow). Lastly, resistance elements are those through which energy dissipation occurs as a function a bond power variable (or, if the model is nonlinear, some combination thereof). See Figure 2 for an example of an interaction between Inertance and Compliance elements in a translational mechanical system.
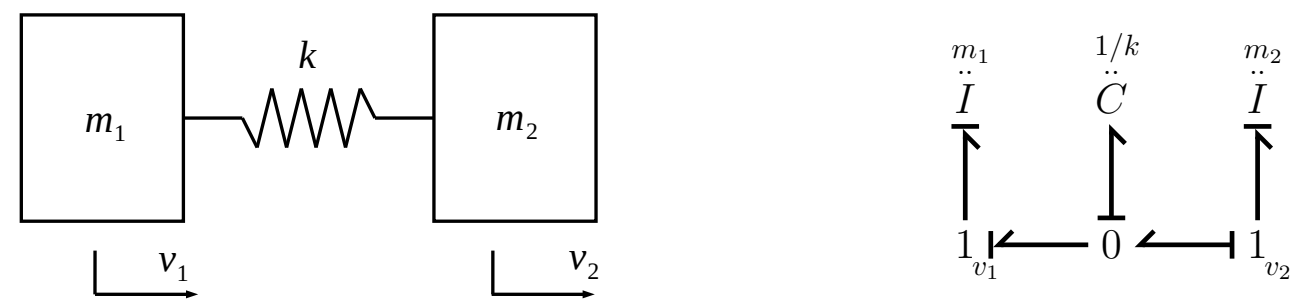

Figure 2: Simple dynamic system and its Bond Graph representation.

Since an element cannot prescribe both power variables to another element, a Bond Graph is said to be causal if all of the causal ticks in the Bond Graph obey this arrangement. If causality is violated, then the Bond Graph is acausal and cannot be said to represent a real physical system. The model developed in this paper is causal, and in "preferred" integral causality, i.e. efforts are functions of displacements for capacitance, and flows are functions of momenta for inertance. In this sense, the Bond Graph of Figure 2 is causal. For more information on non-integral (i.e. derivative) causality, see (Karnopp, Margolis, and Rosenberg 2012).

In order to facilitate interaction of inertance and compliance in mechanical systems, connections between elements must be properly represented in the Bond Graph to constrain allowable physical interactions. For instance, if two translational masses are connected by a spring as in Figure 2, the force exerted by the spring is a function of the relative displacement between the masses, while the instantaneous velocities of the masses are a function of their respective absolute momenta. To represent this interaction, multi-port "0-" and "1-junctions" are introduced. These multi-port elements can have any number of bonds attached to them, and represent physical connections between elements. 1-junctions have the properties that the flows of all bonds attached to them are equal, and the sum of all efforts, following power convention, is zero. 
Therefore, 1 -junctions are used to represent unique velocities of interest in the system. 0 -junctions prescribe that all efforts on all bonds connected to it are equal, and that the sum of all flows (again, following power convention) is zero. 0-junctions are thus used as summing nodes for flows in the bond graph of mechanical systems. Referring to Figure 2, the bond graph properly represents the interaction between the spring and the two masses.

The next features to describe are so-called two-port elements, transformers (TF) and gyrators (GY). These are through-power elements that do not dissipate or store energy. The primary purpose of these elements is to convert between energy domains (in this paper, the energy domain conversions are limited to being between rotational and translational mechanical domains). Transformers obey the constitutive laws $e_{1}=m e_{2}$ and $m f_{1}=f_{2}$, where $m$ is a modulus parameter selected by the modeler to properly represent the physical system being described. Gyrators obey the relation $e_{2}=r f_{1}$ and $e_{1}=r f_{2}$, where $r$ is the gyrator modulus. In the model to be developed, transformers will be used to represent cross product terms inherent in relative velocities between rotating bodies, and gyrators represent cross-product terms that appear when taking the time-derivative of momentum vectors in a rotating from. Transformers and gyrators may be appended by an M (MTF or MGY), indicating that the modulus may be a time-varying or non-constant parameter.

The last features needed to develop this Bond Graph model are sources. There are only two kinds of sources: effort sources and flow sources, represented in the Bond Graph as SE and SF, respectively. These elements represent exogenous inputs to the system, which may be desired inputs, controlled process inputs, or disturbances. For example, the force on an object in a gravity field can be represented by an effort source, and the vertical velocity profile that is input to the bottom of a tire in a traditional quarter-car model would be represented by a flow source.

A feature to notice is that $\mathrm{R}, \mathrm{C}$, and I elements may contain a colon followed by a parameter next to their symbol in the Bond Graph. This indicates that the constitutive law governing the element is linear, with the fixed parameter as labeled indicating the proportionality constant. Transformers, gyrators, and sources always contain a similar labeling system, but in these cases do not indicate anything about the modeling assumptions made (such as linearity), and merely shows the variable used for the respective input or modulus.

A massive benefit of Bond Graphs is their modularity. For instance, the Bond Graphs of sub-systems can be developed independently, and then attached using relevant bonds. For mechanical systems, unique velocities can be modeled equal across several subsystems by forming a bond between the relevant 1 -junctions of each Bond Graph. Then, causality allows the modeler to trace the effect to see exactly how the added dynamics propogate to the state equations.

This has been a concise description of Bond Graph modeling techniques. Like the physical systems they represent, Bond Graph modeling can be highly nuanced, and more details can be found in Reference (Karnopp, Margolis, and Rosenberg 2012).

\section{CTBA MODEL DEVELOPMENT}

A diagram of the system being modeled is presented in Figure 3, where only the right-side variables and coordinates are shown for clarity. The left side variables must simply be translated along relevant axes, and respective subscripts changed.

\subsection{Assumptions and constitutive laws}

The first of three constitutive laws from solid mechanics that will be used to model the trailing arm motions is that relative angular displacements in the body-fixed $y$ direction result in a torsion in the beam along the 


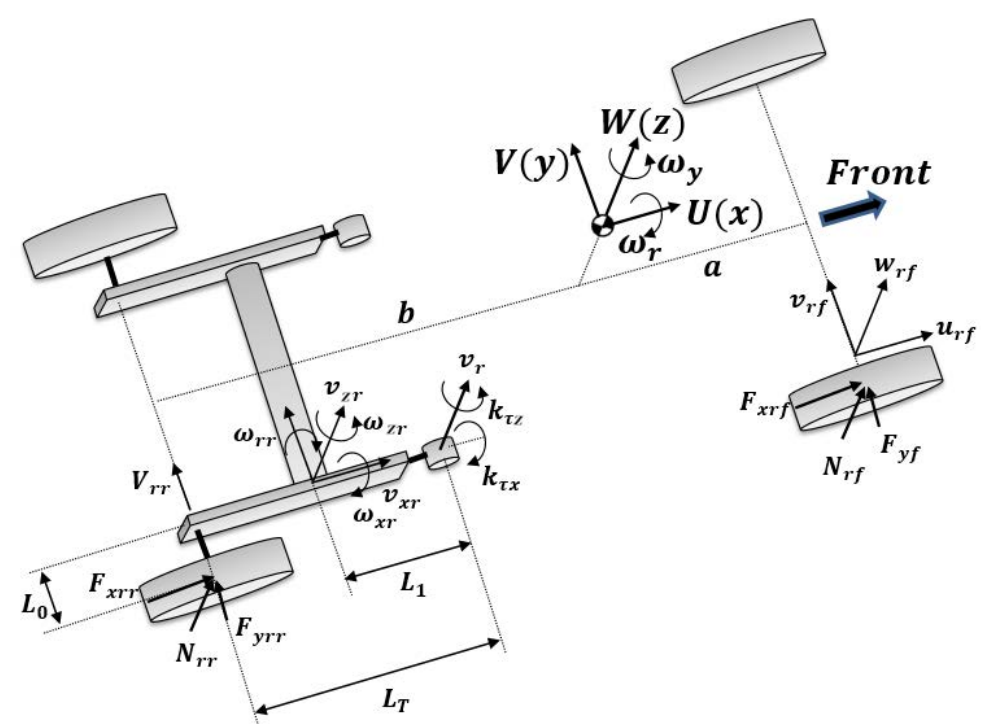

Figure 3: System diagram of full vehicle. The body-fixed $x, y, z$ coordinate system is indicated in parentheses.

same axis. From first principles, a relationship between twist and torque can be written as $\tau=\frac{J G q}{L}$, where $q$ is the difference in angular displacements in the trailing arms, $G$ is the material modulus of rigidity, $L$ is the beam length, and $J$ is the cross-sectional area moment of inertia. Assuming the material properties $J$ and $G$ are constant throughout the cross-section, torque is a linear function of $q$. This is represented as a compliance element in the Bond Graph of Figure 5, with $k_{\tau r b}=\frac{J G}{L_{r b}}$ (See table 1 for subscript descriptions) (Beer, Johnston, and Dewolf 2002).

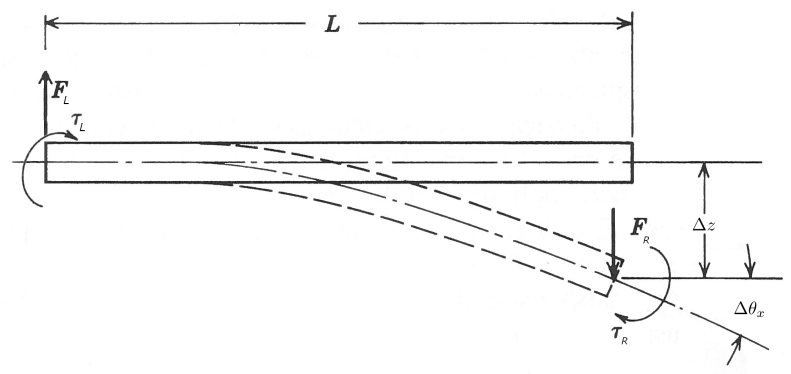

Figure 4: Plane motion of a cantilever beam (Karnopp, Margolis, and Rosenberg 2012).

The second relationship between the trailing arms is the planar motion that occurs between the ends of the torsion beam. It is assumed that the beam behaves like cantilever beam in solid mechanics. Figure 4 shows such a beam. Given a displacement of the right end of Figure 4 in either translation or rotation, a reaction force and moment occur. This reaction force-moment pair results in the generalized symmetric stiffness matrix Equation 1, whose elements are a function of beam properties (Karnopp, Margolis, and Rosenberg 2012).

$$
\left[\begin{array}{l}
F \\
\tau
\end{array}\right]=\left[\begin{array}{ll}
k_{11} & k_{12} \\
k_{12} & k_{22}
\end{array}\right]\left[\begin{array}{c}
\Delta z \\
\Delta \theta x
\end{array}\right]=E I\left[\begin{array}{cc}
12 / L^{3} & -6 / L^{2} \\
-6 / L^{2} & 4 / L
\end{array}\right]\left[\begin{array}{c}
\Delta z \\
\Delta \theta
\end{array}\right] .
$$

This is represented in Bond Graphs by a multiport compliance element with two bonds. In the current case, the reactive force and moment at the opposite end result in 4-port compliance elements, labeled as primary and secondary modes in Figure 5. These derive from the equations relating relative displacements 
between the ends of the beam. With reference to the dimensions of Figure 4, these are $\Delta \theta_{x}=\theta_{x l}-\theta_{x l}$ and $\Delta z=z_{z l}-\left(z_{z r}+L \theta_{x l}\right)$. After some manipulation, these relative displacements can be transformed using equation 1 into a $4 \times 4$ stiffness matrix relating planar displacements at the left and right end of the beam,

$$
\left[\begin{array}{c}
F_{z l} \\
F_{z r} \\
\tau_{x l} \\
\tau_{x r}
\end{array}\right]=E I\left[\begin{array}{cccc}
-12 / L^{3} & 12 / L^{3} & 6 / L^{2} & 6 / L^{2} \\
12 / L^{3} & -12 / L^{3} & -6 / L^{2} & -6 / L^{2} \\
6 / L^{2} & -6 / L^{2} & -4 / L & -2 / L \\
6 / L^{2} & -6 / L^{2} & -2 / L & -4 / L
\end{array}\right]\left[\begin{array}{c}
z_{z l} \\
z_{z r} \\
\theta_{x l} \\
\theta_{x r}
\end{array}\right]
$$

The third relationship is exactly the same as the second, but constrains motions in the perpendicular plane ( $x-y$ plane (secondary bending) instead of $y-z$ plane (primary bending)). In addition, planar compliance of the CTBA occur independent of the state of displacements in the orthogonal plane. These primary and secondary bending modes, along with the beam axial twist, properly constrain relative motion between the left and right trailing arms.

The model assumptions for the sprung mass and front suspension are relatively standard. The sprung mass has 5 degrees of freedom (no pitch). The front suspension is vertical. All dampers and springs are assumed linear elements. The unsprung masses have vertical momenta independent of the sprung mass. The front suspension is assumed to contain a linear spring and damper at each corner, and remain perpendicular to the ground; this is a linearized kinematic approximation, often called "vertical suspension" in vehicle dynamics modeling. Tire deflections are assumed linear functions of vertical tire displacement. An anti-roll bar exerts equal and opposite forces on the sprung masses, and the force is proportional to the difference between each unsprung mass displacement relative to the body. The vehicle inputs will include vertical input velocities at each of the four corners, front steer angle, and gravity. Torque, representing brakes or in-wheel motors, can be applied between the rear trailing arms and the wheels, which have rotational inertia. The wheels are also fixed to rotate with their respective trailing arms, and have diametral rotational inertias in those directions. Tires longitudinal and lateral forces are linearly related with slip ratio and slip angle, respectively. The CTBA acts as the rear anti-roll bar.

\subsection{The Bond Graph model}

Figure 6 contains the Bond Graph for the front suspension. The Bond Graph representation of the sprung mass is shown in Figure 7. The triangular shape derives from the coupled velocity terms that result from transforming forces and moments from a rotating to an inertial reference frame (also known as gyroscopic coupling). This is necessary since forces at the contact patch are defined with respect to the body.

Also included in Figure 7 are the lateral and longitudinal tire forces generated at the front tire contact patches. Notice that the lateral forces are represented as a single term. This comes from the fact that linearization eliminates any distinction between the left and right contact patches (Karnopp 2013). Lateral tire forces are defined as $F_{\text {lat }}=C_{\alpha} \alpha$, which is a linear tire where $C_{\alpha}$ is assumed constant. Longitudinal tire forces are defined as $F_{\text {long }}=\mu s N$, where $\mu$ is a function of the road-tire interface (assumed constant for this paper), $N$ is the tire normal force, and $s$ is the slip ratio, $s=\frac{R \omega-U}{|U|}$ (See Table 1 and 2 for descriptions of parameters and variables). Also, since the front tire lateral force is for the entire axle, the axle force is $F_{y f}=2 C_{\alpha} \alpha_{f}$. 


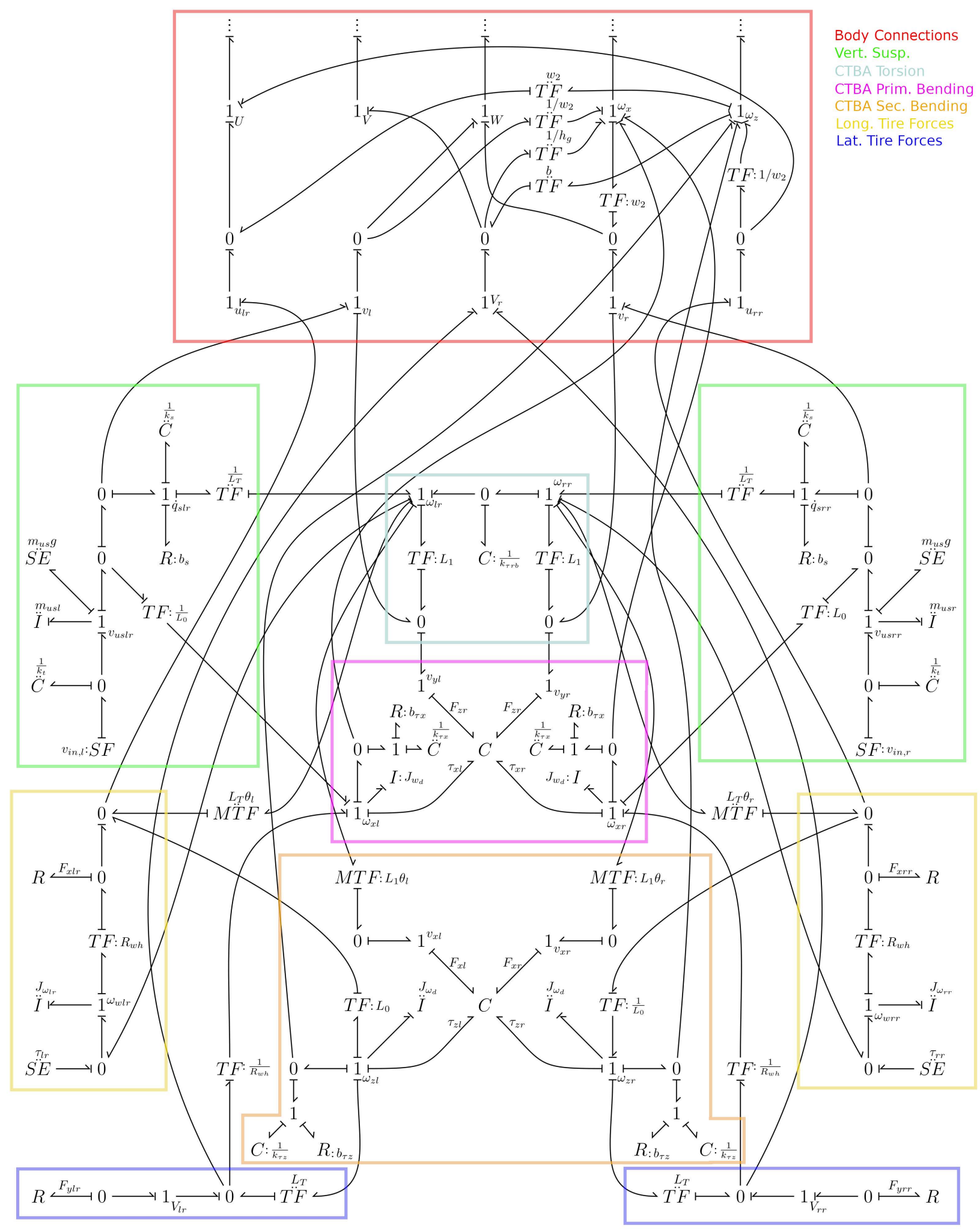

Figure 5: Bond Graph representation of the rear half of a CTBA-equipped vehicle. Colored boxes enclose relevant system subsections. 
The slip angles, $\alpha$, are defined for the front axle and each rear tire as

$$
\begin{aligned}
& \alpha_{f}=\frac{V+h_{g} \omega_{x}+a \omega_{z}}{U}+\delta \\
& \alpha_{r r}=\frac{-V-R_{w h} \omega_{x r}+L_{T} \omega_{y r}}{U+w_{2} \omega_{z}} \\
& \alpha_{l r}=\frac{-V-R_{w h} \omega_{x l}+L_{T} \omega_{y l}}{U-w_{2} \omega_{z}} .
\end{aligned}
$$

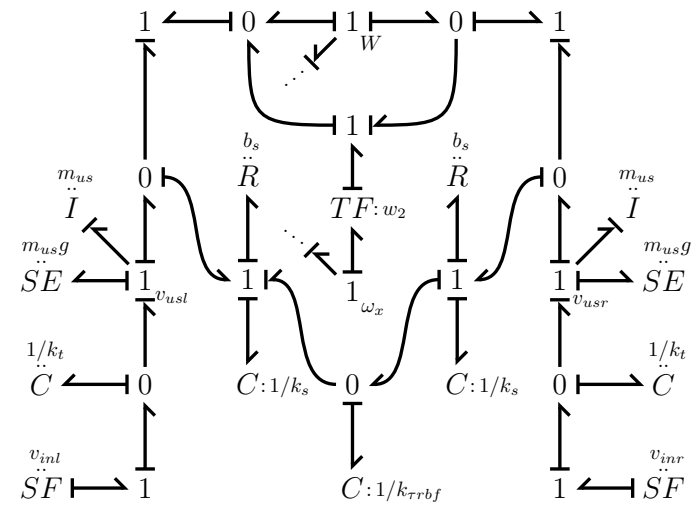

Figure 6: Bond Graph of front suspension.

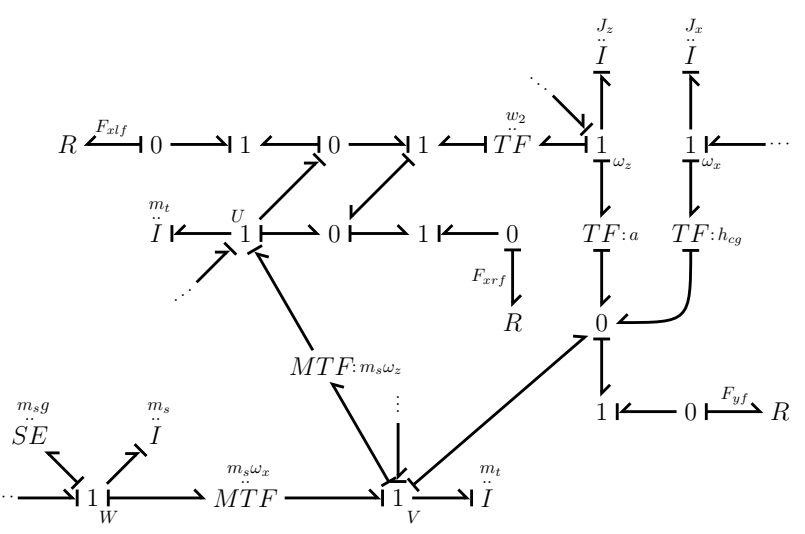

Figure 7: Bond Graph of sprung mass.

After following the methods of Section 2, the Bond Graph in Figure 5 can be generated from the system diagram of Figure 3 and related modeling assumptions. As described earlier, this Bond Graph is a subsystem of the vehicle, and when the CG velocity bonds (those with ellipses) are attached to the Bond Graphs of Figures 6 and 7, the complete vehicle Bond Graph is formed.

From Figure 5, with all energy storage elements are in integral causality, the explicit set of first order state equations that is generated following standard Bond Graph techniques is in the Appendix. Note that there is no pitch degree of freedom allowed for the sprung mass. It was decided that pitch dynamics would have negligible effects on global behavior for this study.

\section{STATIC AND DYNAMIC TEST CONDITIONS}

The suite of tests to which the vehicle model will be subjected comprise of static and dynamic tests. The static tests are common tests performed on real vehicles by automobile manufactures using a Suspension Parameter Measurement Device (SPMD). These tests are left and right brake lock (longitudinal force test), rear axle lateral force, vehicle roll test, and a vertical wheel displacement condition (bump test). Some results are shown from SPMD tests and from the Bond Graph model subjected to equivalent tests in simulation.

The dynamic test will be an initial forward velocity (straight line trajectory) at normal road speeds followed by a step left steer input of front wheels. This test will demonstrate the dynamic capabilities of the model, and some common outputs generally of interest to the vehicle dynamicis are shown. The step steer and bump will demonstrate that the simulation can reveal transient and steady state dynamics in the model. Vehicle forward speed is set at constant $65 \mathrm{kph}$, after which a step steer of $3^{\circ}$ is introduced. 


\section{RESULTS}

The static tests for both model simulation and SPMD are shown in Figures 8. The Bond Graph model behaves similarly to SPMD on-vehicle tests. Note that the simulation results do not follow the SPMD results exactly in all cases, but exhibit a similar trend (slope direction).

Lastly, the dynamic test was performed. A few of the relevant outputs from the test are visible in Figure 9.
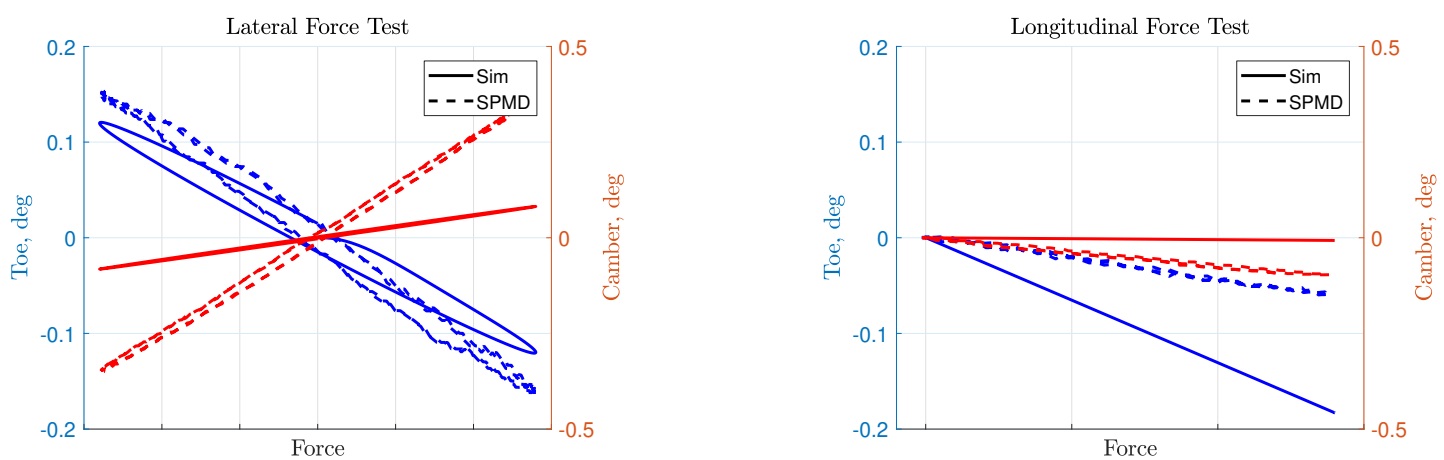

Figure 8: SPMD and simulation results for lateral and longitudinal force condition (force units removed for proprietary purposes).
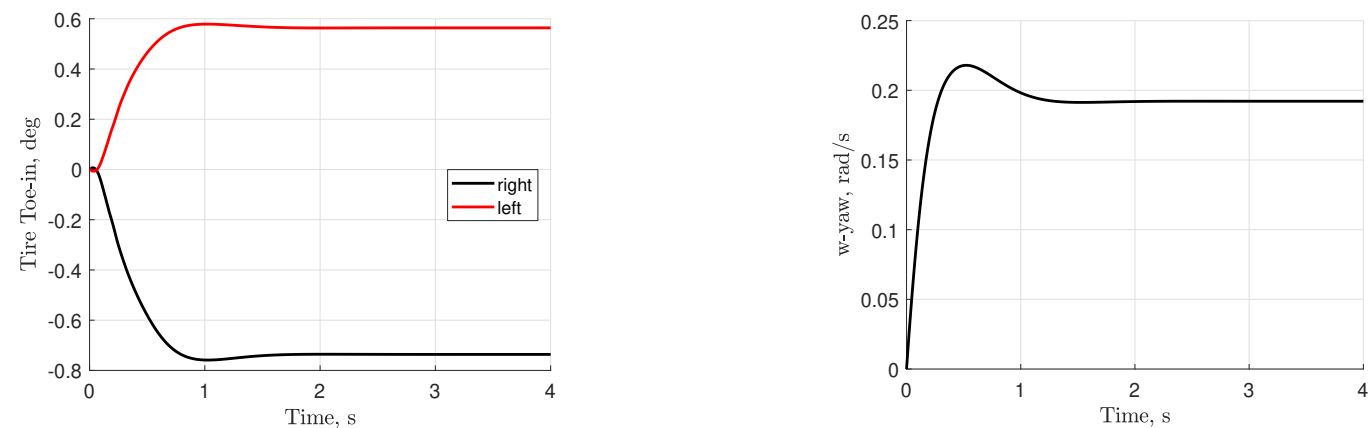

Figure 9: Some simulation results for dynamic test.

\section{CONCLUSION}

A lower-order systems model developed from first principles can suggest trends of higher order models and real physical systems. The model generated using Bond Graphs produced results that trend similarly with the actual vehicle SPMD data. The similarity suggest the viability of lower-order dynamic models in supplanting FEM as the early indicator of vehicle performance in the design process. Further in the design process, the low-order model parameters can be updated using data from higher order models, such as FEM, for verification of global vehicle behavior and continued testing in simulation.

\section{ACKNOWLEDGMENTS}

The authors would like to thank Hyundai Motor Company for its generous contributions in facilitating positive interactions between the faculty and graduate student researchers at UC Davis and the engineers of Hyundai. 
McCrone, Margolis and Kim

\section{A APPENDICES}

Dynamic system equations:

$$
\begin{aligned}
& \dot{p}_{u s l f}=k_{t} q_{t l f}-k_{s} q_{s l f}-b_{s} \dot{q}_{s l f}+k_{\tau r b f} q_{\tau r b f}-m_{u s} g \\
& \dot{p}_{u s r f}=k_{t} q_{t r f}-k_{s} q_{s r f}-b_{s} \dot{q}_{s r f}-k_{\tau r b f} q_{\tau r b f}-m_{u s} g \\
& \dot{p}_{u s l r}=k_{t} q_{t l r}-k_{s} q_{s l r}-b_{s} \dot{q}_{s l r}-\theta_{l} F_{x l r}-\frac{1}{L_{T}}\left(\tau_{l r}-L_{1} \theta_{l} F_{x l}-L_{1} F_{z l}-k_{\tau r b} q_{\tau r b}\right)-m_{u s} g \\
& \dot{p}_{u s r r}=k_{t} q_{t r r}-k_{s} q_{s r r}-b_{s} \dot{q}_{s r r}-\theta_{r} F_{x r r}-\frac{1}{L_{T}}\left(\tau_{r r}+L_{1} \theta_{r} F_{x r}+L_{1} F_{z r}+k_{\tau r b} q_{\tau r b}\right)-m_{u s} g \\
& \dot{p}_{\omega x l}=-\tau_{x l}+R_{w h} F_{y l r}-k_{\tau x} q_{\tau x l}+L_{0} k_{s} q_{s l r}+L_{0} b_{s} \dot{q}_{s l r} \\
& +\frac{L_{0}}{L_{t}}\left(\tau_{l r}+L_{T} \theta_{l} F_{x l r}+L_{1} \theta_{l} F_{x l}+L_{1} F_{z l}-k_{\tau r b} q_{\tau r b}\right)-b_{\tau x} \dot{q}_{\tau x l} \\
& \dot{p}_{\omega x r}=-\tau_{x r}+R_{w h} F_{y r r}-k_{\tau x} q_{\tau x r}-L_{0} k_{s} q_{s r r}-L_{0} b_{s} \dot{q}_{s r r} \\
& -\frac{L_{0}}{L_{t}}\left(\tau_{r r}+k_{\tau r b} q_{\tau r b}+L_{1} \theta_{r} F_{x r}+L_{1} F_{z r}+L_{t} \theta_{r} F_{x r r}\right)-b_{\tau x} \dot{q}_{\tau x r} \\
& \dot{p}_{\omega y l}=-\tau_{z l}-L_{T} F_{y l r}-k_{\tau y} q_{\tau y l}-b_{\tau y} \dot{q}_{\tau z l}-L_{0} F_{x l r} \\
& \dot{p}_{\omega y r}=-\tau_{z r}-L_{T} F_{y r r}-k_{\tau y} q_{\tau y r}-b_{\tau y} \dot{q}_{\tau z r}+L_{0} F_{x r r} \\
& \dot{p}_{\omega l r}=\tau_{l r}-R_{w h} F_{x l r} \\
& \dot{p}_{\omega r r}=\tau_{r r}-R_{w h} F_{x r r} \\
& \dot{p}_{U}=m_{t} \omega_{z} V+F_{x l r}+F_{x r r}+F_{x l f}+F_{x r f} \\
& \dot{p}_{W}=-m_{s} g-m_{t} \omega_{x} V+k_{s}\left(q_{s l r}+q_{s r r}+q_{s l f}+q_{s r f}\right)+b_{s}\left(\dot{q}_{s l r}+\dot{q}_{s r r}+\dot{q}_{s l f}+\dot{q}_{s r f}\right) \\
& +\frac{1}{L_{T}}\left(\tau_{l r}+L_{t} \theta_{l} F_{x l r}+L_{1} \theta_{l} F_{x l}+L_{1} F_{z l}-k_{\tau r b} q_{\tau r b}+\tau_{r r}+k_{\tau r b} q_{\tau r b}+L_{1} \theta_{r} F_{x r}+L_{1} F_{z r}+L_{t} \theta_{r} F_{x r r}\right) \\
& \dot{p}_{V}=m_{s} \omega_{x} W-m_{t} \omega_{z} U+F_{y f}+F_{y l r}+F_{y r r} \\
& \dot{p}_{\omega_{x}}=w_{2}\left(k_{s r} q_{s l r}+b_{s r} \dot{q}_{s l r}+F_{z l}-k_{s r} q_{s r r}-b_{s r} \dot{q}_{s r r}-F_{z r}+\frac{1}{L_{t}}\left(\tau_{l r}+L_{t} \theta_{l} F_{x l r}+L_{1} \theta_{l} F_{x l}+L_{1} F_{z l}\right.\right. \\
& \left.\left.-k_{\tau r b} q_{\tau r b}-\tau_{r r}-k_{\tau r b} q_{\tau r b}-L_{1} \theta_{r} F_{x r}-L_{1} F_{z r}-L_{t} \theta_{r} F_{x r r}\right)\right)+h_{g}\left(F_{y l r}+F_{y r r}\right)+b_{\tau x} \dot{q}_{\tau x l} \\
& +k_{\tau x} q_{\tau x l}-\tau_{x l}+b_{\tau x} \dot{q}_{\tau x r}+k_{\tau x} q_{\tau x r}-\tau_{x r}-t_{f}\left(-k_{s} q_{s l f}-b_{s} \dot{q}_{s l f}+2 k_{\tau r b f} q_{\tau r b f}+b_{s} \dot{q}_{s r f}+k_{s} q_{s r f}\right. \\
& \dot{p}_{\omega z}=a F_{y f}+w 2\left(F_{x r f}-F_{x l f}\right)+w_{2}\left(F_{x r r}-F_{x l r}\right)-b\left(F_{y l r}+F_{y r r}\right)+k_{\tau y} q_{\tau y l}+b_{\tau y} \dot{q}_{\tau z l}+\tau_{z l}+k_{\tau y} q_{\tau y r} \\
& +b_{\tau y} \dot{q}_{\tau z r}+\tau_{z r} \\
& \begin{aligned}
\dot{q}_{s l r} & =v_{u s l r}-L_{0} \omega_{x l}-W-w_{2} \omega_{x} & \dot{q}_{z r} & =\frac{L_{1}}{L_{t}} \dot{q}_{s r r}+W-w_{2} \omega_{x} \\
\dot{q}_{s r r} & =v_{u s r r}+L_{0} \omega_{x r}-W+w_{2} \omega_{x} & \dot{q}_{x l} & =\frac{L_{1}}{L_{t}} \theta_{l} \dot{q}_{s l r} \\
\dot{q}_{s l f} & =\frac{p_{u s l f}}{m_{u s}}-W-w_{2} \omega_{x} & \dot{q}_{x r} & =\frac{L_{1}}{L_{t}} \theta_{r} \dot{q}_{s r r} \\
\dot{q}_{s r f} & =\frac{p_{u s r f}}{m_{u s}}-W+w_{2} \omega_{x} & \dot{q}_{\tau z l} & =\frac{p_{\omega y l}}{J_{w d}}-\omega_{z} \\
\dot{q}_{t l r} & =v_{i l r}-\frac{p_{u s l r}}{m_{u s}} & \dot{q}_{\tau z r} & =\frac{p_{\omega y r}}{J_{w d}}-\omega_{z}
\end{aligned}
\end{aligned}
$$




$$
\begin{aligned}
\dot{q}_{t r r} & =v_{i r r}-\frac{p_{u s r r}}{m_{u s}} & \dot{q}_{\tau x l} & =\frac{p_{\omega x l}}{J_{w d}}-\omega_{x} \\
\dot{q}_{\tau r b} & =\frac{1}{L_{T}}\left(\dot{q}_{s r r}-\dot{q}_{s l r}\right) & \dot{q}_{\tau x r} & =\frac{p_{\omega x r}}{J_{w d}}-\omega_{x} \\
q_{\tau r b f} & =\dot{q}_{s r f}-\dot{q}_{s l f} & \dot{q}_{t l f} & =v_{i l f}-\frac{p_{u s l f}}{m_{u s}} \\
\dot{q}_{z l} & =\frac{L_{1}}{L_{t}} \dot{q}_{s l r}+W+w_{2} \omega_{x} & \dot{q}_{t r f} & =v_{i r f}-\frac{p_{u s r f}}{m_{u s}} .
\end{aligned}
$$

Table 1: Descriptions of parameter constants used throughout the paper.

\begin{tabular}{rll}
\hline Parameter & Units & Description \\
\hline$k_{\tau r b f}$ & $\mathrm{Nm} / \mathrm{rad}$ & front roll bar stiffness \\
$k_{\tau r b}$ & $\mathrm{Nm} / \mathrm{rad}$ & rear roll bar stiffness \\
$k_{s\{f, r\}}$ & $\mathrm{N} / \mathrm{m}$ & front,rear suspension stiffness \\
$k_{t}$ & $\mathrm{~N} / \mathrm{m}$ & tire stiffness \\
$k_{\# \#}$ & $\mathrm{~N} / \mathrm{m}$ & \#\# are numeric indices to elements in planar beam stiffness matrix of Equation 2 \\
$k_{\tau x}$ & $\mathrm{Nm} / \mathrm{rad}$ & rotational bushing stiffness of each trailing arm about body-fixed $x$ axis \\
$k_{\tau y}$ & $\mathrm{Nm} / \mathrm{rad}$ & rotational bushing stiffness of each trailing arm about body-fixed $y$ axis \\
$b_{s\{f, r\}}$ & $\mathrm{N} \mathrm{s} / \mathrm{m}$ & front,rear suspension damping coefficient \\
$b_{\tau x}$ & $\mathrm{Nms} / \mathrm{rad}$ & rotational bushing damping coefficient of each trailing arm about body-fixed $x$ axis \\
$b_{\tau y}$ & $\mathrm{Nms} / \mathrm{rad}$ & rotational bushing damping coefficient of each trailing arm about body-fixed $y$ axis \\
$m_{s}$ & $\mathrm{~kg}$ & mass of the sprung mass \\
$m_{t}$ & $\mathrm{~kg}$ & total mass of the vehicle \\
$m_{u s}$ & $\mathrm{~kg}$ & unsprung mass at each of the 4 corners \\
$J_{\omega d}$ & $\mathrm{~kg} \mathrm{~m} / \mathrm{s}^{2}$ & tire diametral moment of inertia \\
$J_{\omega\{l, r\}}$ & $\mathrm{kg} \mathrm{m} / \mathrm{s}^{2}$ & \{left,right $\}$ tire rotational moment of inertia \\
$R_{w h}$ & $\mathrm{~m}$ & tire radius \\
$L_{1}$ & $\mathrm{~m}$ & distance along trailing arm from sprung mass connection bushing to torsion beam \\
$L_{T}$ & $\mathrm{~m}$ & total trailing arm length from sprung mass connection bushing to wheel center \\
$L_{0}$ & $\mathrm{~m}$ & distance from trailing arm to wheel center measured along wheel rotation axis \\
$a$ & $\mathrm{~m}$ & distance from sprung mass center of gravity (CG) to front axle \\
$b$ & $\mathrm{~m}$ & $x$-distance from sprung mass CG to trailing arm bushing \\
$w_{2}$ & $\mathrm{~m}$ & half the track width $(y$-distance between left and right tire contact patches) \\
$h_{g}$ & $\mathrm{~m}$ & $z$-distance from wheel center to sprung mass CG \\
$t_{f}$ & $\mathrm{~m}$ & front and rear half track \\
$g$ & $\mathrm{~m} / \mathrm{s}^{2}$ & acceleration due to gravity \\
\hline & &
\end{tabular}


Table 2: Descriptions of variables used throughout the paper.

\begin{tabular}{|c|c|c|}
\hline Variable & Units & Description \\
\hline$p_{\{U, V, W\}}$ & $\mathrm{kg} \mathrm{m} / \mathrm{s}$ & $\{$ longitudinal $(x)$, lateral $(y), \operatorname{vertical}(z)\}$ momentum of sprung mass CG \\
\hline$p_{\omega\{x, z\}}$ & $\mathrm{kg} \mathrm{rad} / \mathrm{s}$ & angular $\{x, z\}$-momentum of sprung mass \\
\hline$p_{u s\{l f, r f, l r, r r\}}$ & $\mathrm{kg} \mathrm{m} / \mathrm{s}$ & vertical momentum of $\{$ left front,...,right rear $\}$ sprung mass $C G$ \\
\hline$p_{\omega\{x, y\}\{l, r\}}$ & $\mathrm{kgrad} / \mathrm{s}$ & angular $\{x, y\}$-momentum of $\{$ left,right $\}$ tire \\
\hline$p_{J_{\omega\{l, r\}}}$ & $\mathrm{kg} \mathrm{rad} / \mathrm{s}$ & axial angular momentum of $\{$ left,right $\}$ tire \\
\hline$q_{t}\{l f, r f, l r, r r\}$ & $\mathrm{m}$ & $\{$ left front,,.., right rear $\}$ tire displacement \\
\hline$q_{s}\{l f, r f, l r, r r\}$ & $\mathrm{m}$ & $\{$ left front,...,right rear $\}$ suspension displacement \\
\hline$q_{\tau\{x, z\}\{l, r\}}$ & $\mathrm{rad}$ & angular $\{x, y\}$-displacement of $\{$ left,right $\}$ trailing arm relative to vehicle \\
\hline$q_{\{x, z\}\{l, r\}}$ & $\mathrm{m}$ & relative displacement of left and right trailing arms in $\{x, y\}$ \\
\hline$q_{\tau r b\{f, r\}}$ & $\mathrm{rad}$ & $\{$ front,rear $\}$ roll bar angular displacement \\
\hline$F_{x\{l f, r f, l r, r r\}}$ & $\mathrm{N}$ & longitudinal tire forces at $\{$ left front,...,right rear $\}$ contact patch \\
\hline$F_{y\{f, l r, r r\}}$ & $\mathrm{N}$ & lateral forces at $\{$ front axle, left rear tire, right rear tire $\}$ \\
\hline$v_{\text {in },\{l f, r f, l r, r r\}}$ & $\mathrm{m} / \mathrm{s}$ & vertical road input to $\{$ left front,,.., right rear $\}$ tire \\
\hline$\tau_{\{l r, r r\}}$ & $\mathrm{Nm}$ & wheel torque input to $\{$ left rear, right rear $\}$ wheel \\
\hline$F_{\{x, z\}\{l, r\}}$ & $\mathrm{N}$ & force on trailing arms from torsion beam \\
\hline$\tau_{\{x, z\}\{l, r\}}$ & $\mathrm{Nm}$ & torque on trailing arms from torsion beam \\
\hline$\omega_{\{x, z\}}$ & $\mathrm{rad} / \mathrm{s}$ & sprung mass angular velocity about $\{x, z\}$ \\
\hline$\{U, V, W\}$ & $\mathrm{m} / \mathrm{s}$ & sprung mass linear velocity along $\{x, y, z\}$ \\
\hline$\theta_{\{l, r\}}$ & $\mathrm{rad}$ & trailing arm angle with respect to vehicle body measured about $y$ \\
\hline$\omega_{\{x, z\}\{l, r\}}$ & $\mathrm{rad} / \mathrm{s}$ & angular $\{x, y\}$-velocity of $\{$ left,right $\}$ trailing arm \\
\hline$\alpha$ & $\mathrm{rad}$ & tire slip ratio \\
\hline$\delta$ & $\mathrm{rad}$ & front axle steer angle \\
\hline
\end{tabular}

\section{REFERENCES}

Beer, F. P., E. Johnston, and J. T. Dewolf. 2002. Mechanics of Materials. 5th. 5th ed. McGraw-Hill.

Crolla, D. A., D. E. Foster, T. Kobayashi, and N. Vaughan. 2015. Encyclopedia of Automotive Engineering: Engines-fundamentals, Volume 1. John Wiley \& Sons.

Karnopp, D. C. 2013. Vehicle Dynamics, Stability, and Control. 2nd ed. CRC Press.

Karnopp, D. C., D. L. Margolis, and R. C. Rosenberg. 2012. System Dynamics. 5th ed. Wiley and Sons.

\section{AUTHOR BIOGRAPHIES}

D. JORDAN MCCRONE is a graduate student researcher in the Hyundai Center for Excellence at UC Davis, a collaborative venture between students and faculty at UC Davis and visiting engineers from Hyundai in Korea. His research interest is in vehicle dynamics with a particular focus on control systems and advanced modeling techniques for multi-energy domain systems. His email is djmccrone@ucdavis.edu.

DONALD MARGOLIS is Professor of Mechanical Engineering at UC Davis. He has extensive experience in teaching system dynamics at the graduate and undergraduate levels and has published numerous papers on the industrial applications of the subject. His email is dlmargolis@ucdavis.edu.

NAMHO KIM is an expert in the modeling and design of CTBA suspensions. His research interests include integrated chassis design, and allocation and control development of in-wheel motors for next-generation EV models. His email is babylonx@ @yundai.com. 